\title{
Unbounded Quasinormal Operators Revisited
}

\author{
Zenon Jan Jabłoński, Il Bong Jung and Jan Stochel
}

\begin{abstract}
Various characterizations of unbounded closed densely defined operators commuting with the spectral measures of their moduli are established. In particular, Kaufman's definition of an unbounded quasinormal operator is shown to coincide with that given by the third-named author and Szafraniec. Examples demonstrating the sharpness of results are constructed.
\end{abstract}

Mathematics Subject Classification (2010). Primary 47B20; Secondary 47B37.

Keywords. Quasinormal operator, paranormal operator, spectral measure, weighted shift on a directed tree.

\section{Introduction}

The class of bounded quasinormal operators was introduced by Brown in [3]. Two different definitions of unbounded quasinormal operators appeared independently in [15] and (a few years later) in [21]. In the present paper we show that both of these definitions coincide (cf. Theorem 3.1). We also discuss the question of whether the equality in Kaufman's definition of quasinormality can be replaced by inclusion. It is shown that the answer is in the affirmative if the inclusion is properly chosen (cf. Theorem 3.1 and Remark 3.2). Next, we characterize quasinormality of unbounded operators in terms of the truncated operator Stieltjes moment problem [cf. Theorem 3.6(iv)]. This part of the paper is inspired by a result of Embry which characterizes quasinormality of bounded operators by means of the operator Stieltjes moment problem (cf. [9, page 63]). Yet another characterization of quasinormality of unbounded operators is given in Theorem 3.6(v). It states that a closed densely defined operator $C$ is quasinormal if and only if the equality $C^{* n} C^{n}=\left(C^{*} C\right)^{n}$ holds for $n=2,3$. In the case of bounded operators, this characterization has been known for specialists working in this area since late 1980s (recently it has

Research of the first and the third authors was supported by the MNiSzW (Ministry of Science and Higher Education) Grant NN201 546438 (2010-2013). Research of the second author was supported by the National Research Foundation of Korea(NRF) grant funded by the Korea government(MSIP) (No. 2009-0083521). 
been published in [14, Proposition 13]; unfortunately, this paper contains several errors). The proof of Theorem 3.6 is essentially more advanced and requires the use of the technique of bounded vectors. That the single equality $C^{* n} C^{n}=\left(C^{*} C\right)^{n}$ does not imply the quasinormality of $C$ is elucidated by Examples 5.1, 5.3 and 5.5. The first example is related to Toeplitz operators on the Hardy space $H^{2}$, while two others are linked to weighted shifts on a directed tree with one branching vertex of valency 2. Example 5.3 enables us to construct a bounded injective non-quasinormal composition operator $C$ on an $L^{2}$ space over a $\sigma$-finite measure space such that $\left(C^{*} C\right)^{2}=C^{* 2} C^{2}$ (cf. Remark 5.4). Example 5.5 shows how to separate (with respect to $n$ ) the classes of operators $C$ satisfying the equality $C^{* n} C^{n}=\left(C^{*} C\right)^{n}$. In Example 5.6 we construct a quasinormal operator $C$ such that $C^{* n} \subsetneq\left(C^{n}\right)^{*}$ for every $n \geqslant 2$. In Sect. 4 we show that closed densely defined operators $C$ which satisfy the equality $C^{* 2} C^{2}=\left(C^{*} C\right)^{2}$ and have closed squares, are paranormal (cf. Theorem 4.1). As a consequence, operators constructed in Examples 5.1, 5.3 and 5.5 are instances of bounded injective paranormal operators which are not hyponormal (see the first paragraph of Sect. 5). The paper concludes with an appendix discussing orthogonal sums of unbounded operators. Moreover, two open problems are formulated (cf. Problems 2.3 and 3.9).

In what follows $\mathbb{N}, \mathbb{Z}_{+}$and $\mathbb{R}_{+}$stand for the sets of positive integers, nonnegative integers and nonnegative real numbers respectively. Denote by $\mathfrak{B}(X)$ the $\sigma$-algebra of all Borel subsets of a topological space $X$. Let $A$ be an operator in a complex Hilbert space $\mathcal{H}$ (all operators considered in this paper are linear). Denote by $\mathcal{D}(A), \mathcal{N}(A), \mathcal{R}(A), A^{*}, \bar{A}$ and $\sigma(A)$ the domain, the kernel, the range, the adjoint, the closure and the spectrum of $A$ (in case they exist) respectively. Set $\mathcal{D}^{\infty}(A)=\bigcap_{n=0}^{\infty} \mathcal{D}\left(A^{n}\right)$. In what follows, we write $C^{* n}$ in place of $\left(C^{*}\right)^{n}$. A vector subspace $\mathcal{E}$ of $\mathcal{D}(A)$ is said to be a core for $A$ if $\mathcal{E}$ is dense in $\mathcal{D}(A)$ with respect to the graph norm of $A$. If $A$ is closed, then $\mathcal{E}$ is a core for $A$ if and only if $A=\overline{\left.A\right|_{\mathcal{E}}}$, where $\left.A\right|_{\mathcal{E}}$ stands for the restriction of $A$ to $\mathcal{E}$. A closed densely defined operator $A$ has a (unique) polar decomposition $A=U|A|$, where $U$ is a partial isometry on $\mathcal{H}$ such that $\mathcal{N}(U)=\mathcal{N}(A)$ and $|A|$ is the square root of $A^{*} A$ (cf. [25, Theorem 7.20]). If $A$ and $B$ are two operators in $\mathcal{H}$ such that the graph of $A$ is contained in the graph of $B$, then we write $A \subseteq B$ or $B \supseteq A$. In what follows, $\boldsymbol{B}(\mathcal{H})$ stands for the $C^{*}$-algebra of all bounded operators $A$ in $\mathcal{H}$ such that $\mathcal{D}(A)=\mathcal{H}$. Denote by $I$ the identity operator on $\mathcal{H}$. Recall that a closed densely defined operator $N$ in $\mathcal{H}$ is normal if $N^{*} N=N N^{*}$. If $N$ is a normal operator, then its spectral measure, denoted here by $E_{N}$ and usually defined on $\mathfrak{B}(\sigma(N))$, can be thought of as a spectral Borel measure on any fixed closed subset of $\mathbb{C}$ containing $\sigma(N)$. In any case, the closed support of such measure coincides with $\sigma(N)$. For this and other facts concerning unbounded operators we refer the reader to $[2,25]$.

\section{Commutativity}

In this section we discuss the question of commutativity of a bounded normal operator with an unbounded closed operator. Though this is a more 
general approach than we need in this paper, the question itself seems to be of independent interest.

Given $\mathcal{F} \subseteq \boldsymbol{B}(\mathcal{H})$, we write $\mathcal{F}^{\prime}=\{T \in \boldsymbol{B}(\mathcal{H}): \forall A \in \mathcal{F}, T A=A T\}$ and $\mathcal{F}^{\prime \prime}=\left(\mathcal{F}^{\prime}\right)^{\prime}$. If $A$ is an operator in $\mathcal{H}$, then we set

$$
\mathfrak{C}_{\mathrm{s}}(A)=\left\{T \in \boldsymbol{B}(\mathcal{H}): T A \subseteq A T \text { and } T^{*} A \subseteq A T^{*}\right\}
$$

Let $N$ be a normal operator in $\mathcal{H}$. For a complex Borel function $\phi$ on $\sigma(N)$, we abbreviate $\int_{\sigma(N)} \phi \mathrm{d} E_{N}$ to $\phi(N)$ (cf. [2]). If $A$ is an operator in $\mathcal{H}$ such that $E_{N}(\Delta) A \subseteq A E_{N}(\Delta)$ for every $\Delta \in \mathfrak{B}(\sigma(N))$, then we write $E_{N} A \subseteq A E_{N}$.

Lemma 2.1. Let $A$ be a closed operator in $\mathcal{H}$ and $N$ be a normal operator in $\mathcal{H}$. Consider the following three conditions:

(i) $N A \subseteq A N$ and $N^{*} A \subseteq A N^{*}$,

(ii) $E_{N} A \subseteq A E_{N}$,

(iii) $\phi(N) A \subseteq A \phi(N)$ for every bounded complex Borel function $\phi$ on $\sigma(N)$.

Then the conditions (ii) and (iii) are equivalent. Moreover, if $N$ is bounded, then the conditions (i), (ii) and (iii) are equivalent.

Proof. Assume (ii) holds. For $h \in \mathcal{H}$, we set $\mu_{h}=\left\langle E_{N}(\cdot) h, h\right\rangle$. Take $f \in \mathcal{D}(A)$. Suppose $\phi$ is a bounded complex Borel function on $\sigma(N)$. Then clearly $\phi \in L^{2}\left(\mu_{f}+\mu_{A f}\right)$. By [19, Theorem 3.13], there exists a sequence $\left\{s_{n}\right\}_{n=1}^{\infty}$ of complex Borel simple functions on $\sigma(N)$ such that $\lim _{n \rightarrow \infty} \int_{\sigma(N)}\left|\phi-s_{n}\right|^{2} \mathrm{~d}\left(\mu_{f}+\mu_{A f}\right)=0$. Hence, we have

$$
\begin{aligned}
\left\|\phi(N) f-s_{n}(N) f\right\|^{2} & =\int_{\sigma(N)}\left|\phi-s_{n}\right|^{2} \mathrm{~d} \mu_{f} \rightarrow 0 \text { as } n \rightarrow \infty, \\
\left\|\phi(N) A f-s_{n}(N) A f\right\|^{2} & =\int_{\sigma(N)}\left|\phi-s_{n}\right|^{2} \mathrm{~d} \mu_{A f} \rightarrow 0 \text { as } n \rightarrow \infty .
\end{aligned}
$$

Since, by our assumption, $s_{n}(N) A \subseteq A s_{n}(N)$ for all $n \geqslant 1$, we deduce that $s_{n}(N) f \rightarrow \phi(N) f$ and $A\left(s_{n}(N) f\right) \rightarrow \phi(N) A f$ as $n \rightarrow \infty$. This and the closedness of $A$ imply (iii). The implication (iii) $\Rightarrow$ (ii) is obvious.

Now suppose $N$ is bounded and (i) holds. Since $A$ is closed, $\mathfrak{C}_{\mathrm{s}}(A)$ is a von Neumann algebra with unit $I$. By assumption $N \in \mathfrak{C}_{\mathrm{s}}(A)$ and thus $\mathfrak{C}_{\mathrm{S}}(A)$ contains the von Neumann algebra $\mathcal{W}^{*}(I, N)$ generated by $I$ and $N$. By von Neumann's double commutant theorem ${ }^{1}, \mathcal{W}^{*}(I, N)=\left\{N, N^{*}\right\}^{\prime \prime}$. Since $E_{N}(\mathfrak{B}(\sigma(N))) \subseteq\left\{N, N^{*}\right\}^{\prime \prime}$, we get (ii). The implication (iii) $\Rightarrow$ (i) is obvious. This completes the proof.

Remark 2.2. Regarding Lemma 2.1, we note that by approximating $\phi$ by polynomials in two complex variables $z$ and $\bar{z}$ and arguing as in the proof of (ii) $\Rightarrow$ (iii), we can show that (i) $\Rightarrow$ (ii). It is worth pointing out that if $A$ is closed and densely defined and $\sigma(A) \neq \mathbb{C}$, then Lemma 2.1 remains true if one drops the assumption that $N^{*} A \subseteq A N^{*}$. Indeed, the inclusion $N A \subseteq A N$ implies that $(\lambda-A)^{-1} N \subseteq N(\lambda-A)^{-1}$ for any fixed $\lambda \in \mathbb{C} \backslash \sigma(A)$, and thus by the Fuglede theorem, for each bounded complex Borel function $\phi$ on $\sigma(N)$,

\footnotetext{
${ }^{1}$ In fact, by the Fuglede theorem $\left\{N, N^{*}\right\}^{\prime \prime}=\{N\}^{\prime \prime}$.
} 
$(\lambda-A)^{-1} \phi(N)=\phi(N)(\lambda-A)^{-1}$, which in turn implies that $\phi(N) A \subseteq A \phi(N)$ (in particular, $N^{*} A \subseteq A N^{*}$ ).

The above remark suggests the following problem which is related to the Fuglede theorem.

Problem 2.3. Let $A$ be a closed operator in $\mathcal{H}$ and $N$ be a bounded normal operator in $\mathcal{H}$ such that $N A \subseteq A N$. Is it true that $N^{*} A \subseteq A N^{*}$ ?

Now we consider the issue of commutativity of an unbounded operator with a spectral measure of a positive selfadjoint operator.

Lemma 2.4. Let $A$ be a closed operator in $\mathcal{H}$ and $R$ be a positive selfadjoint operator in $\mathcal{H}$. Then the following conditions are equivalent:

(i) $E_{R} A \subseteq A E_{R}$,

(ii) $(I+R)^{-1} A \subseteq A(I+R)^{-1}$,

(iii) $\phi(R) A \subseteq A \phi(R)$ for every bounded complex Borel function $\phi$ on $\sigma(R)$.

Proof. (i) $\Rightarrow$ (ii) Apply Lemma 2.1 with $\phi(x)=\frac{1}{1+x}$.

(ii) $\Rightarrow$ (i) Set $N=(I+R)^{-1}$ and note that $N \in \boldsymbol{B}(\mathcal{H})$. Applying Lemma 2.1, we see that $E_{N} A \subseteq A E_{N}$. Since $R$ and $N$ are positive and selfadjoint, we may (and do) regard $E_{R}$ and $E_{N}$ as spectral Borel measures on $\mathbb{R}_{+}$. Let $\phi:[0, \infty) \rightarrow(0,1]$ be the homeomorphism given by $\phi(x)=\frac{1}{1+x}$ for $x \in[0, \infty)$. By [2, Theorem 5.4.10], we have

$$
N=\int_{[0, \infty)} \phi(x) E_{R}(\mathrm{~d} x)=\int_{(0,1]} t E_{R} \circ \phi^{-1}(\mathrm{~d} t),
$$

where $E_{R} \circ \phi^{-1}$ is the spectral measure given by $\left(E_{R} \circ \phi^{-1}\right)(\Delta)=E_{R}\left(\phi^{-1}(\Delta)\right)$ for $\Delta \in \mathfrak{B}((0,1])$. By the uniqueness assertion in the spectral theorem, this implies that $E_{N}(\Delta)=E_{R}\left(\phi^{-1}(\Delta \cap(0,1])\right)$ for all $\Delta \in \mathfrak{B}\left(\mathbb{R}_{+}\right)$, and thus

$$
E_{R}(\Delta)=E_{R}\left(\phi^{-1}(\phi(\Delta))\right)=E_{N}(\phi(\Delta)), \quad \Delta \in \mathfrak{B}\left(\mathbb{R}_{+}\right) .
$$

This and the fact that $E_{N}(\Delta) A \subseteq A E_{N}(\Delta)$ for all $\Delta \in \mathfrak{B}\left(\mathbb{R}_{+}\right)$yield (i).

(i) $\Leftrightarrow$ (iii) Apply Lemma 2.1.

\section{Quasinormality Revisited}

Following [21] (see [3, Lemma 4.1] for the bounded case) we say that a closed densely defined operator $C$ in $\mathcal{H}$ is quasinormal if $C$ commutes with $E_{|C|}$, i.e., $E_{|C|} C \subseteq C E_{|C|}$. By [21, Proposition 1], a closed densely defined operator $C$ in $\mathcal{H}$ is quasinormal if and only if $U|C| \subseteq|C| U$, where $C=U|C|$ is the polar decomposition of $C$. It is well-known that quasinormal operators are always subnormal and that the reverse implication does not hold in general. For more information on quasinormal operators we refer the reader to [3,7], the bounded case, and to $[21,16]$, the unbounded one. Our aim in this section is to show that the above definition of quasinormality coincide with the one given by Kaufman in [15] [see condition (ii) below]. In fact, we prove that the equality in Kaufman's definition can be replaced by inclusion (see condition (i) below). Recall that if $C$ is a closed densely defined operator in $\mathcal{H}$, then $C^{*} C$ is a positive selfadjoint operator (cf. [2, Section 4.5]). 
Theorem 3.1. Let $C$ be a closed densely defined operator in $\mathcal{H}$. Then the following conditions are equivalent:

(i) $C C^{*} C \subseteq C^{*} C C$,

(ii) $C C^{*} C=C^{*} C C$,

(iii) $\left(I+C^{*} C\right)^{-1} C \subseteq C\left(I+C^{*} C\right)^{-1}$,

(iv) $E_{|C|} C \subseteq C E_{|C|}$.

Proof. (i) $\Rightarrow$ (iii) It follows from (i) that $C\left(I+C^{*} C\right) \subseteq\left(I+C^{*} C\right) C$. This implies that $\left(I+C^{*} C\right)^{-1} C\left(I+C^{*} C\right) \subseteq C$, which yields (iii).

(iii) $\Rightarrow$ (iv) Apply $[2$, Theorem 5.4.10] and Lemma 2.4 to the function $\phi(x)=\chi_{\Delta}(\sqrt{x})$, where $\chi_{\Delta}$ is the characteristic function of a set $\Delta \in \mathfrak{B}\left(\mathbb{R}_{+}\right)$.

(iv) $\Rightarrow$ (ii) Let $C=U|C|$ be the polar decomposition of $C$. By [21, Proposition 1] and [18, Lemma 2.2], we have $U|C|=|C| U$, which implies that

$$
C C^{*} C=U|C|^{3}=|C|^{2} U|C|=C^{*} C C .
$$

(ii) $\Rightarrow$ (i) Evident.

Remark 3.2. It is worth mentioning that the inclusion $C^{*} C C \subseteq C C^{*} C$, which is opposite to the one in Theorem 3.1(i), do not imply quasinormality. To see this, take a nonzero closed densely defined operator $C$ such that $\mathcal{D}\left(C^{2}\right)=\{0\}$ (see $[17,5]$ for the case of symmetric operators, or $[4,13]$ for the case of hyponormal composition operators). Then $\mathcal{D}\left(C^{*} C C\right)=\{0\}$ and thus $C^{*} C C \subseteq C C^{*} C$. However, $C$ is not quasinormal. Indeed, otherwise, by Theorem 3.1, $C^{*} C C=C C^{*} C=U|C|^{3}$, which implies that $C^{*} C C$ is densely defined, a contradiction.

To prove Theorem 3.6, which is one of the main results of this paper, we need three preparatory lemmas.

Lemma 3.3. If $C$ is a closed densely defined operator in $\mathcal{H}$, then $\mathcal{H}_{\infty} \subseteq$ $\mathcal{D}^{\infty}\left(C^{*} C\right)$ and $\left(I+C^{*} C\right) \mathcal{H}_{\infty}=\mathcal{H}_{\infty}$, where $\mathcal{H}_{\infty}=\bigcup_{n=1}^{\infty} \mathcal{R}\left(E_{|C|}([0, n])\right)$.

Proof. Set $R=C^{*} C$ and $\mathcal{H}_{n}=\mathcal{R}\left(E_{|C|}([0, n])\right)$ for $n \in \mathbb{N}$. Fix $n \in \mathbb{N}$. It is clear that $\mathcal{H}_{n}=\overline{\mathcal{H}}_{n}$ and $\mathcal{H}_{n} \subseteq \mathcal{D}(R)$. By [2, Theorem 6.3.2], the space $\mathcal{H}_{n}$ reduces $R$, and thus $\mathcal{H}_{n} \subseteq \mathcal{D}^{\infty}(R)$. By the closed graph theorem (or via [2, Chapter 5]), the operator $R_{n}:=\left.R\right|_{\mathcal{H}_{n}}$ is bounded. Since $R_{n}$ is positive, we deduce that $\left(I+R_{n}\right)^{-1} \in \boldsymbol{B}\left(\mathcal{H}_{n}\right)$ and consequently $(I+R) \mathcal{H}_{n}=\mathcal{R}\left(I+R_{n}\right)=$ $\mathcal{H}_{n}$. This completes the proof.

Lemma 3.4. If $C$ is a closed densely defined operator in $\mathcal{H}$ and $n \in \mathbb{Z}_{+}$, then

(i) $\left(C^{*} C\right)^{n} \subseteq C^{* n} C^{n}$ if and only if $\left(C^{*} C\right)^{n}=C^{* n} C^{n}$,

(ii) $\left(C^{*} C\right)^{n} \subseteq\left(C^{n}\right)^{*} C^{n}$ if and only if $\left(C^{*} C\right)^{n}=\left(C^{n}\right)^{*} C^{n}$ provided $C^{n}$ is densely defined,

(iii) if $\left(C^{*} C\right)^{n}=C^{* n} C^{n}$, then $\left(C^{*} C\right)^{n}=\left(C^{n}\right)^{*} C^{n}$.

Proof. (iii) Observe that the operator $\left(C^{*} C\right)^{n}$ is selfadjoint (see e.g., [25, Theorems 7.14 and 7.19]). Since $\left(C^{*} C\right)^{n}=C^{* n} C^{n}$, we see that $C^{n}$ is densely defined and consequently $\left(C^{*} C\right)^{n} \subseteq\left(C^{n}\right)^{*} C^{n}$. As selfadjoint operators are maximal symmetric and $\left(C^{n}\right)^{*} C^{n}$ is symmetric, we get $\left(C^{*} C\right)^{n}=\left(C^{n}\right)^{*} C^{n}$.

Similar argument can be used to prove (i) and (ii). 
Lemma 3.5. If $C$ is a quasinormal operator in $\mathcal{H}$, then

$$
\left(C^{*} C\right)^{n}=C^{* n} C^{n}=\left(C^{n}\right)^{*} C^{n}, \quad n \in \mathbb{Z}_{+} .
$$

Proof. Let $C=U|C|$ be the polar decomposition of $C$. By [18, Lemma 2.2], we have $U|C|=|C| U$. First we show that

$$
|C|^{k}=U^{*}|C|^{k} U, \quad k \in \mathbb{N} .
$$

Indeed, it follows from $U|C|=|C| U$ that $U|C|^{k}=|C|^{k} U$ for all $k \in \mathbb{N}$. Since $U^{*} U$ is the orthogonal projection of $\mathcal{H}$ onto $\overline{\mathcal{R}(|C|)}$, we get $|C|^{k}=U^{*} U|C|^{k}=$ $U^{*}|C|^{k} U$ for all $k \in \mathbb{N}$, which yields (3.2).

Using induction on $n \geqslant 1$, we will show that the left-hand equality in (3.1) holds. Clearly it is valid for $n=1$. Assuming it holds for a fixed $n \geqslant 1$ and noting that $(U|C|)^{*}=|C| U^{*}$, we get

$$
\begin{aligned}
C^{*(n+1)} C^{n+1} & =C^{*}\left(C^{* n} C^{n}\right) C=C^{*}\left(C^{*} C\right)^{n} C=(U|C|)^{*}|C|^{2 n} U|C| \\
& =|C|\left(U^{*}|C|^{2 n} U\right)|C| \stackrel{(3.2)}{=}|C||C|^{2 n}|C|=\left(C^{*} C\right)^{n+1},
\end{aligned}
$$

which proves our claim. Applying Lemma 3.4(iii) completes the proof.

Now we are ready to prove the afore-mentioned characterization of quasinormal operators. In the case of bounded operators the equivalences (i) $\Leftrightarrow($ ii $) \Leftrightarrow$ (iii) have been proved by Embry in [9, page 63$]$, and the implication $(\mathrm{v}) \Rightarrow(\mathrm{i})$ can be found in [14].

Theorem 3.6. Let $C$ be a closed densely defined operator in $\mathcal{H}$. Then the following conditions are equivalent:

(i) $C$ is quasinormal,

(ii) $C^{* n} C^{n}=\left(C^{*} C\right)^{n}$ for every $n \in \mathbb{Z}_{+}$,

(iii) there exists a (unique) spectral Borel measure $E$ on $\mathbb{R}_{+}$such that

$$
C^{* n} C^{n}=\int_{\mathbb{R}_{+}} x^{n} E(\mathrm{~d} x), \quad n \in \mathbb{Z}_{+},
$$

(iv) there exists a (unique) spectral Borel measure $E$ on $\mathbb{R}_{+}$such that

$$
C^{* n} C^{n}=\int_{\mathbb{R}_{+}} x^{n} E(\mathrm{~d} x), \quad n \in\{1,2,3\},
$$

(v) $C^{* n} C^{n}=\left(C^{*} C\right)^{n}$ for $n \in\{2,3\}$.

Before proving Theorem 3.6 we make a remark.

Remark 3.7. By Lemma 3.4(i), Theorem 3.6 remains valid if the equality in (ii) and (v) is replaced by the inclusion " $\supseteq$ ". This is not the case for " $\subseteq$ ". To see this, it is enough to consider a nonzero closed densely defined operator $C$ such that $\mathcal{D}\left(C^{2}\right)=\{0\}$ (consult Remark 3.2).

Proof of Theorem 3.6. Note that the uniqueness of $E$ in (iii) and (iv) follows from the equality $C^{*} C=\int_{\mathbb{R}_{+}} x E(\mathrm{~d} x)$ and the spectral theorem. In both cases $E=E_{C^{*} C}$.

(i) $\Rightarrow$ (ii) Apply Lemma 3.5. 
(ii) $\Rightarrow$ (iii) By the multiplicative property of spectral integral (cf. [25, Theorem 7.19]), $E=E_{C^{*} C}$ meets our requirements.

(iii) $\Rightarrow$ (iv) Evident.

(iv) $\Rightarrow$ (v) This follows from the multiplicative property of spectral integral and the equality $E=E_{C{ }^{*} C}$.

$(\mathrm{v}) \Rightarrow(\mathrm{i})$ By (v), we have

$$
\begin{aligned}
& C^{* 2} C C^{*} C^{2}=C^{*}\left(C^{*} C\right)^{2} C=C^{* 3} C^{3}=\left(C^{*} C\right)^{3}, \\
& C^{*} C C^{* 2} C^{2}=\left(C^{*} C\right)^{3}, \quad C^{* 2} C^{2} C^{*} C=\left(C^{*} C\right)^{3} .
\end{aligned}
$$

The penultimate equality yields $\mathcal{D}\left(\left(C^{*} C\right)^{3}\right) \subseteq \mathcal{D}\left(C^{*} C^{2}-C C^{*} C\right)$. Thus, we have

$$
\begin{aligned}
\left\|\left(C^{*} C^{2}-C C^{*} C\right) f\right\|^{2} & =\left\langle C^{* 2} C C^{*} C^{2} f, f\right\rangle-\left\langle C^{*} C C^{* 2} C^{2} f, f\right\rangle \\
& -\left\langle C^{* 2} C^{2} C^{*} C f, f\right\rangle+\left\langle\left(C^{*} C\right)^{3} f, f\right\rangle \stackrel{(3.3)}{=} 0
\end{aligned}
$$

for every $f \in \mathcal{D}\left(\left(C^{*} C\right)^{3}\right)$, which implies that

$$
C^{*} C^{2} f=C C^{*} C f, \quad f \in \mathcal{D}\left(\left(C^{*} C\right)^{3}\right) .
$$

Therefore this leads to

$$
C\left(I+C^{*} C\right) f=\left(I+C^{*} C\right) C f, \quad f \in \mathcal{D}\left(\left(C^{*} C\right)^{3}\right) .
$$

Since $I+C^{*} C$ is injective and $\left(I+C^{*} C\right)^{-1} \in \boldsymbol{B}(\mathcal{H})$, we deduce that

$$
\begin{aligned}
\left(I+C^{*} C\right)^{-1} C\left(I+C^{*} C\right) f & =C f \\
& =C\left(I+C^{*} C\right)^{-1}\left(I+C^{*} C\right) f, \quad f \in \mathcal{D}\left(\left(C^{*} C\right)^{3}\right) .
\end{aligned}
$$

Applying Lemma 3.3, we obtain

$$
\left(I+C^{*} C\right)^{-1}\left(\left.C\right|_{\mathcal{H}_{\infty}}\right)=\left.C\left(I+C^{*} C\right)^{-1}\right|_{\mathcal{H}_{\infty}} \subseteq C\left(I+C^{*} C\right)^{-1} .
$$

It is well-known that $\mathcal{H}_{\infty}$ is a core for $|C|$ (see e.g., [23, Proposition 4]). Since the domains of $C$ and $|C|$ are equal and the graph norms of $C$ and $|C|$ coincide, we deduce that $\mathcal{H}_{\infty}$ is a core for $C$. This combined with (3.4) yields

$$
\left(I+C^{*} C\right)^{-1} C=\left(I+C^{*} C\right)^{-1} \overline{\left.C\right|_{\mathcal{H}_{\infty}}} \subseteq C\left(I+C^{*} C\right)^{-1} .
$$

By Theorem 3.1 this completes the proof.

The following corollary can be viewed as a special case of $[18$, Proposition 9.1]. Our proof is different, less involved and much shorter.

Corollary 3.8. If $C$ is a quasinormal operator, then for every $n \in \mathbb{Z}_{+}, C^{n}$ is a quasinormal operator.

Proof. Since quasinormal operators are subnormal, we infer from [20, Proposition 5.3.] that $C^{n}$ is closed. By [21, Proposition 5], $C^{n}$ is densely defined. Fix $k \in \mathbb{Z}_{+}$. It follows from Theorem 3.6 that $\left(C^{n}\right)^{* k}\left(C^{n}\right)^{k} \supseteq C^{* n k} C^{n k}=$ $\left(C^{*} C\right)^{n k}$. Since $\left(C^{n}\right)^{* k}\left(C^{n}\right)^{k}$ is symmetric and $\left(C^{*} C\right)^{n k}$ is selfadjoint, we get

$$
\left(C^{n}\right)^{* k}\left(C^{n}\right)^{k}=\left(C^{*} C\right)^{n k} .
$$

Similarly, we see that $\left(\left(C^{n}\right)^{*} C^{n}\right)^{k} \supseteq\left(C^{* n} C^{n}\right)^{k}=\left(C^{*} C\right)^{n k}$. Since the operator $\left(\left(C^{n}\right)^{*} C^{n}\right)^{k}$ is symmetric (in fact selfadjoint) and the operator $\left(C^{*} C\right)^{n k}$ 
is selfadjoint, we get $\left(\left(C^{n}\right)^{*} C^{n}\right)^{k}=\left(C^{*} C\right)^{n k}$. This, (3.5) and Theorem 3.6 imply that $C^{n}$ is quasinormal.

In view of Lemma 3.5 and Theorem 3.6, the following problem arises.

Problem 3.9. Is it true that, if $C$ is a closed operator in $\mathcal{H}$ such that $C^{3}$ is densely defined and ${ }^{2}\left(C^{*} C\right)^{n}=\left(C^{n}\right)^{*} C^{n}$ for $n \in\{2,3\}$, then $C$ is quasinormal?

\section{More on $\left(C^{*} C\right)^{2}=C^{* 2} C^{2}$}

Regarding Theorem 3.6, it is tempting to ask the question whether the condition (v) can be replaced by the single equality $\left(C^{*} C\right)^{2}=C^{* 2} C^{2}$ without affecting the conclusion. Though, the answer is in the negative (cf. Sect. 5), operators satisfying this equality are often paranormal (see Theorem 4.1 and Corollary 4.2 below). Recall that an operator $C$ in $\mathcal{H}$ is said to be paranormal if $\|C f\|^{2} \leqslant\left\|C^{2} f\right\|\|f\|$ for all $f \in \mathcal{D}\left(C^{2}\right)$. We refer the reader to [8] for examples of unbounded paranormal operators with pathological properties related to closability (in particular, paranormal operators may not be closable).

Theorem 4.1. Let $C$ be a closed densely defined operator in $\mathcal{H}$ such that

$$
\left(C^{*} C\right)^{2}=C^{* 2} C^{2} \text {. }
$$

Then the following assertions hold:

(i) $\mathcal{D}\left(|C|^{2}\right) \subseteq \mathcal{D}\left(C^{2}\right)$, the operator $\left.C^{2}\right|_{\mathcal{D}\left(|C|^{2}\right)}$ is closed and $\mathcal{D}\left(|C|^{4}\right)$ is a

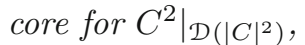

(ii) $\|C f\|^{2} \leqslant\left\|C^{2} f\right\|\|f\|$ for all $f \in \mathcal{D}\left(|C|^{2}\right)$,

(iii) $C^{2}$ is closed if and only if $\mathcal{D}\left(C^{2}\right) \subseteq \mathcal{D}\left(|C|^{2}\right)$,

(iv) $C$ is paranormal if and only if $C^{2}$ is closed.

Proof. It follows from (4.1) that $\mathcal{D}\left(|C|^{4}\right) \subseteq \mathcal{D}\left(C^{2}\right)$. First we show that the operator $\left.C^{2}\right|_{\mathcal{D}\left(|C|^{4}\right)}$ is closable and

$$
\mathcal{D}\left(|C|^{2}\right)=\mathcal{D}\left(\overline{\left.C^{2}\right|_{\mathcal{D}\left(|C|^{4}\right)}}\right) .
$$

Indeed, it follows from (4.1) that

$$
\left\|\left.|C|^{2}\right|_{\mathcal{D}\left(|C|^{4}\right)} f\right\|=\left\|\left.C^{2}\right|_{\mathcal{D}\left(|C|^{4}\right)} f\right\| \text { for all } f \in \mathcal{D}\left(|C|^{4}\right) .
$$

Since $|C|^{2}$ is closed, the operator $\left.|C|^{2}\right|_{\mathcal{D}\left(|C|^{4}\right)}$ is closable. This and (4.3) imply that $\left.C^{2}\right|_{\mathcal{D}\left(|C|^{4}\right)}$ is closable. Applying (4.3) again and the fact that $\mathcal{D}\left(|C|^{4}\right)$ is a core for $|C|^{2}$ (see e.g., [2, Theorem 4.5.1]), we get (4.2).

Continuing the proof, we deduce from the Cauchy-Schwarz inequality that

$$
\|C f\|^{2}=\left\langle|C|^{2} f, f\right\rangle \leqslant\left\||C|^{2} f\right\|\|f\| \stackrel{(4.3)}{=}\left\|C^{2} f\right\|\|f\|, \quad f \in \mathcal{D}\left(|C|^{4}\right) .
$$

Take $f \in \mathcal{D}\left(|C|^{2}\right)$. Since $\mathcal{D}\left(|C|^{4}\right)$ is a core for $|C|^{2}$, there exists a sequence $\left\{f_{n}\right\}_{n=1}^{\infty} \subseteq \mathcal{D}\left(|C|^{4}\right)$ such that $f_{n} \rightarrow f$ and $|C|^{2} f_{n} \rightarrow|C|^{2} f$ as $n \rightarrow \infty$. Since,

\footnotetext{
${ }^{2}$ See also Lemma 3.4(ii).
} 
by (4.3), $\left\|C^{2}\left(f_{n}-f_{m}\right)\right\|=\left\||C|^{2}\left(f_{n}-f_{m}\right)\right\|$ for all $m, n \in \mathbb{N}$, we deduce that there exists $h \in \mathcal{H}$ such that

$$
C^{2} f_{n} \rightarrow h \text { as } n \rightarrow \infty .
$$

It follows from (4.4) that

$$
\left\|C\left(f_{n}-f_{m}\right)\right\|^{2} \leqslant\left\|C^{2}\left(f_{n}-f_{m}\right)\right\|\left\|f_{n}-f_{m}\right\|, \quad m, n \in \mathbb{N},
$$

which implies that there exists $g \in \mathcal{H}$ such that $C f_{n} \rightarrow g$ as $n \rightarrow \infty$. Since $C$ is closed and $f_{n} \rightarrow f$ as $n \rightarrow \infty$, we see that $f \in \mathcal{D}(C)$ and $C f_{n} \rightarrow C f$ as $n \rightarrow \infty$. This combined with (4.5) implies that $f \in \mathcal{D}\left(C^{2}\right)$ (hence $\mathcal{D}\left(|C|^{2}\right) \subseteq$ $\mathcal{D}\left(C^{2}\right)$ ) and $C^{2} f_{n} \rightarrow C^{2} f$ as $n \rightarrow \infty$. Since, by (4.4), $\left\|C f_{n}\right\|^{2} \leqslant\left\|C^{2} f_{n}\right\|\left\|f_{n}\right\|$ for all $n \in \mathbb{N}$, we deduce that (ii) holds. Moreover, because $f_{n} \rightarrow f$ and $\left.C^{2}\right|_{\mathcal{D}\left(|C|^{4}\right)} f_{n} \rightarrow C^{2} f$ as $n \rightarrow \infty$, and $\left.C^{2}\right|_{\mathcal{D}\left(|C|^{4}\right)}$ is closable, we conclude that $f \in \mathcal{D}\left(\overline{\left.C^{2}\right|_{\mathcal{D}\left(|C|^{4}\right)}}\right)$ and $C^{2} f=\overline{\left.C^{2}\right|_{\mathcal{D}\left(|C|^{4}\right)}} f$. This means that $\left.C^{2}\right|_{\mathcal{D}\left(|C|^{2}\right)} \subseteq$ $\overline{\left.C^{2}\right|_{\mathcal{D}\left(|C|^{4}\right)}}$. Now, by $(4.2),\left.C^{2}\right|_{\mathcal{D}\left(|C|^{2}\right)}=\overline{\left.C^{2}\right|_{\mathcal{D}\left(|C|^{4}\right)}}$. This completes the proof of the assertions (i) and (ii).

(iii) If $C^{2}$ is closed, then (4.1) and Lemma 3.4(iii) imply that $|C|^{4}=$ $\left|C^{2}\right|^{2}$ and consequently $|C|^{2}=\left|C^{2}\right|$, which yields $\mathcal{D}\left(C^{2}\right)=\mathcal{D}\left(\left|C^{2}\right|\right)=\mathcal{D}\left(|C|^{2}\right)$ (cf. [2, Lemma 8.1.1]). The reverse implication follows from (i).

(iv) If $C$ is paranormal, then, by [22, Proposition 6(iv)], $C^{2}$ is closed. The reverse implication is a direct consequence of (ii) and (iii).

Corollary 4.2. Let $C \in \boldsymbol{B}(\mathcal{H})$. If $\left(C^{*} C\right)^{2}=C^{* 2} C^{2}$, then $C$ is paranormal.

Recall that the spectral radius and the norm of a bounded paranormal operator coincide (cf. [10, Theorem 1]). Note also that the converse implication in Corollary 4.2 does not hold in general. To see this consider a bounded weighted shift with a nonconstant monotonically increasing sequence of positive weights.

Corollary 4.3. If $C \in \boldsymbol{B}(\mathcal{H})$ is either compact or algebraic, then $C$ is normal if and only if $\left(C^{*} C\right)^{2}=C^{* 2} C^{2}$. In particular, if $\mathcal{H}$ is finite dimensional, then this equivalence holds for every operator $C$ on $\mathcal{H}$.

Proof. Apply [6, Theorem 6.5] in the case of algebraic operators, [10, Theorem $2]$ in the case of compact operators, and Corollary 4.2 in both cases.

\section{Examples}

We begin by showing that the equality (4.1) does not imply the quasinormality of $C$. In view of Corollary 4.3, such an operator cannot be constructed in a finite dimensional space. It is worth mentioning that a hyponormal operator $C \in \boldsymbol{B}(\mathcal{H})$ which satisfies the equality (4.1) must be quasinormal (cf. [9, page $63])$. This means that a non-quasinormal operator $C \in \boldsymbol{B}(\mathcal{H})$ which satisfies (4.1) is never hyponormal (though, it is always paranormal, cf. Corollary 4.2). The first counterexample we present is related to Toeplitz operators. We refer the reader to [1] and [7] for more information on Toeplitz operators. 
Example 5.1. Let $S$ be the Hardy shift on the Hardy space $H^{2}$. Let $T_{\varphi}$ be a Toeplitz operator on $H^{2}$ with a symbol $\varphi \in L^{\infty}$ such that $\varphi>0$ almost everywhere with respect to the normalized Lebesgue measure on the unit circle and $\int_{0}^{2 \pi} \varphi\left(\mathrm{e}^{\mathrm{i} t}\right) \mathrm{e}^{\mathrm{i} t} \mathrm{~d} t \neq 0$. Then $T_{\varphi}$ is a bounded injective positive operator and $S$ is an isometry which does not commute with $T_{\varphi}$ (because $\left\langle S T_{\varphi} 1,1\right\rangle=0$ and $\left.\left\langle T_{\varphi} S 1,1\right\rangle \neq 0\right)$. This implies that the operator $C:=$ $S T_{\varphi}^{1 / 2}$ is not quasinormal. Remembering that $S^{*} T_{\varphi} S=T_{\varphi}$ (cf. [1, Proposition 4.2.3]), we get

$$
C^{* 2} C^{2}=T_{\varphi}^{1 / 2} S^{*} T_{\varphi}^{1 / 2} S^{*} S T_{\varphi}^{1 / 2} S T_{\varphi}^{1 / 2}=T_{\varphi}^{1 / 2} S^{*} T_{\varphi} S T_{\varphi}^{1 / 2}=T_{\varphi}^{2}=\left(C^{*} C\right)^{2} .
$$

Before turning to the next example, we note that a (unilateral or bilateral) weighted shift $W$ which satisfies the equality $\left(W^{*} W\right)^{2}=W^{* 2} W^{2}$ is quasinormal. Going a step further, we can verify that there is no bounded nonquasinormal injective weighted shift $W$ which satisfies the following equality

$$
\left(W^{*} W\right)^{3}=W^{* 3} W^{3}
$$

(the details are left to the reader). Passing to more general operators, called weighted shifts on directed trees, we are able to construct bounded nonquasinormal injective operators which satisfy (4.1) [or even (5.1)]. First, we recall necessary definitions and state an auxiliary result which is of some independent interest in itself (cf. Proposition 5.2).

Suppose $\mathscr{T}=(V, E)$ is a directed tree $(V$ and $E$ are the sets of vertices and edges of $\mathscr{T}$, respectively). If $\mathscr{T}$ has a root, we denote it by root. Put $V^{\circ}=V \backslash\{$ root $\}$ if $\mathscr{T}$ has a root and $V^{\circ}=V$ otherwise. For every $u \in V^{\circ}$, there exists a unique $v \in V$, denoted by $\operatorname{par}(u)$, such that $(v, u) \in E$. The Hilbert space of square summable complex functions on $V$ equipped with the standard inner product is denoted by $\ell^{2}(V)$. For $u \in V$, we define $e_{u} \in \ell^{2}(V)$ to be the characteristic function of the one-point set $\{u\}$. Given a system $\boldsymbol{\lambda}=\left\{\lambda_{v}\right\}_{v \in V^{\circ}}$ of complex numbers, we define the operator $S_{\boldsymbol{\lambda}}$ in $\ell^{2}(V)$, which is called a weighted shift on $\mathscr{T}$ with weights $\boldsymbol{\lambda}$, as follows

$$
\mathcal{D}\left(S_{\boldsymbol{\lambda}}\right)=\left\{f \in \ell^{2}(V): \Lambda_{\mathscr{T}} f \in \ell^{2}(V)\right\} \quad \text { and } \quad S_{\boldsymbol{\lambda}} f=\Lambda_{\mathscr{T}} f \text { for } f \in \mathcal{D}\left(S_{\boldsymbol{\lambda}}\right),
$$

where

$$
\left(\Lambda_{\mathscr{T}} f\right)(v)=\left\{\begin{array}{ll}
\lambda_{v} \cdot f(\operatorname{par}(v)) & \text { if } v \in V^{\circ}, \\
0 & \text { otherwise, }
\end{array} \quad v \in V, f \in \mathbb{C}^{V} .\right.
$$

We refer the reader to [11] for more on weighted shifts on directed trees.

Now, we characterize bounded weighted shifts on directed trees satisfying the equality $\left(S_{\boldsymbol{\lambda}}^{*} S_{\boldsymbol{\lambda}}\right)^{n}=S_{\boldsymbol{\lambda}}^{* n} S_{\boldsymbol{\lambda}}^{n}$ for a fixed $n \in \mathbb{Z}_{+}$.

Proposition 5.2. Let $n \in \mathbb{Z}_{+}$. If $S_{\boldsymbol{\lambda}} \in \boldsymbol{B}\left(\ell^{2}(V)\right)$ is a weighted shift on a directed tree $\mathscr{T}=(V, E)$ with weights $\boldsymbol{\lambda}=\left\{\lambda_{v}\right\}_{v \in V^{\circ}}$, then the following two conditions are equivalent:

(i) $\left(S_{\boldsymbol{\lambda}}^{*} S_{\boldsymbol{\lambda}}\right)^{n}=S_{\boldsymbol{\lambda}}^{* n} S_{\boldsymbol{\lambda}}^{n}$,

(ii) $\left\|S_{\lambda} e_{u}\right\|^{n}=\left\|S_{\lambda}^{n} e_{u}\right\|$ for all $u \in V$.

Proof. By the polarization identity, (i) holds if and only if $\left\|\left|S_{\boldsymbol{\lambda}}\right|^{n} f\right\|^{2}=$ $\left\|S_{\lambda}^{n} f\right\|^{2}$ for all $f \in \ell^{2}(V)$. Hence an application of [11, Proposition 3.4.3(iv)] and $[12$, Theorem 3.2.2(ii)] completes the proof. 
The example below deals with weighted shifts on leafless directed trees with one branching vertex of valency 2 (cf. [11, page 67]).

Example 5.3. Fix $\kappa \in \mathbb{Z}_{+} \sqcup\{\infty\}$. Let $\mathscr{T}_{2, \kappa}=\left(V_{2, \kappa}, E_{2, \kappa}\right)$ be the directed tree with $V_{2, \kappa}=\left\{-k: k \in J_{\kappa}\right\} \sqcup\{0\} \sqcup\left\{(i, j): i \in J_{2}, j \in \mathbb{N}\right\}$ and

$$
\begin{aligned}
& E_{2, \kappa}=\left\{(-k,-k+1): k \in J_{\kappa}\right\} \sqcup\left\{(0,(i, 1)): i \in J_{2}\right\} \\
& \sqcup\left\{((i, j),(i, j+1)): i \in J_{2}, j \in \mathbb{N}\right\},
\end{aligned}
$$

where $J_{\iota}=\{k \in \mathbb{N}: k \leqslant \iota\}$ for $\iota \in \mathbb{Z}_{+} \sqcup\{\infty\}$ (the symbol " $\sqcup$ " denotes disjoint union of sets). Take $\alpha_{1}, \alpha_{2} \in \mathbb{C} \backslash\{0\}$ such that $\left|\alpha_{1}\right|^{2}+\left|\alpha_{2}\right|^{2}=1$. Let $\beta_{1}, \beta_{2} \in \mathbb{C} \backslash\{0\}$ be such that $\left|\alpha_{1} \beta_{1}\right|^{2}+\left|\alpha_{2} \beta_{2}\right|^{2}=1$ and $\left(1-\left|\beta_{1}\right|\right)\left(1-\left|\beta_{2}\right|\right) \neq 0$ (clearly, such $\beta_{1}$ and $\beta_{2}$ exist). Define the system of weights $\boldsymbol{\lambda}=\left\{\lambda_{v}\right\}_{v \in V_{2, \kappa}^{\circ}}$ by

$$
\lambda_{v}=\left\{\begin{array}{lll}
\alpha_{i} & \text { if } v=(i, 1) & \text { with } i \in J_{2}, \\
\beta_{i} & \text { if } v=(i, j) \\
1 & \text { otherwise. } & \text { with } i \in J_{2} \text { and } j \geqslant 2,
\end{array}\right.
$$

Let $S_{\boldsymbol{\lambda}}$ be the weighted shift on $\mathscr{T}_{2, \kappa}$ with weights $\boldsymbol{\lambda}$. By [11, Proposition 3.1.8], we see that $S_{\boldsymbol{\lambda}} \in \boldsymbol{B}\left(\ell^{2}\left(V_{2, \kappa}\right)\right)$. It follows from [11, Proposition 8.1.7(ii)] (applied to $u=0$ ) that $S_{\boldsymbol{\lambda}}$ is not quasinormal. However, by Proposition 5.2, $\left(S_{\boldsymbol{\lambda}}^{*} S_{\boldsymbol{\lambda}}\right)^{2}=S_{\boldsymbol{\lambda}}^{* 2} S_{\boldsymbol{\lambda}}^{2}$.

Remark 5.4. It follows from Example 5.3 and [12, Lemma 4.3.1] that there exists a bounded injective non-quasinormal composition operator $C$ on an $L^{2}$ space over a $\sigma$-finite measure space such that $\left(C^{*} C\right)^{2}=C^{* 2} C^{2}$.

Modifying Example 5.3, we will show that for every integer $n \geqslant 2$, there exists a non-quasinormal weighted shift $S_{\boldsymbol{\lambda}} \in \boldsymbol{B}\left(\ell^{2}\left(V_{2,0}\right)\right)$ on $\mathscr{T}_{2,0}$ such that

$$
\left(S_{\boldsymbol{\lambda}}^{*} S_{\boldsymbol{\lambda}}\right)^{n}=S_{\boldsymbol{\lambda}}^{* n} S_{\boldsymbol{\lambda}}^{n} \text { and }\left(S_{\boldsymbol{\lambda}}^{*} S_{\boldsymbol{\lambda}}\right)^{k} \neq S_{\boldsymbol{\lambda}}^{* k} S_{\boldsymbol{\lambda}}^{k} \text { for all } k \in\{2,3, \ldots\} \backslash\{n\} .
$$

Example 5.5. Take $\alpha_{1}, \alpha_{2}, \beta_{1}, \beta_{2} \in \mathbb{C} \backslash\{0\}$ such that $\left|\alpha_{1}\right|^{2}+\left|\alpha_{2}\right|^{2}=1$. Define the system of weights $\boldsymbol{\lambda}=\left\{\lambda_{v}\right\}_{v \in V_{2,0}^{\circ}}$ by

$$
\lambda_{v}=\left\{\begin{array}{lll}
\alpha_{i} & \text { if } v=(i, 1) & \text { with } i \in J_{2}, \\
\beta_{i} & \text { if } v=(i, j) & \text { with } i \in J_{2} \text { and } j \geqslant 2 .
\end{array}\right.
$$

Let $S_{\boldsymbol{\lambda}}$ be the weighted shift on $\mathscr{T}_{2,0}$ with weights $\boldsymbol{\lambda}$. By [11, Proposition 3.1.8], $S_{\boldsymbol{\lambda}} \in \boldsymbol{B}\left(\ell^{2}\left(V_{2,0}\right)\right)$. In view of Proposition 5.2, for every integer $l \geqslant 2$, $\left(S_{\boldsymbol{\lambda}}^{*} S_{\boldsymbol{\lambda}}\right)^{l}=S_{\boldsymbol{\lambda}}^{* l} S_{\boldsymbol{\lambda}}^{l}$ if and only if $\left|\alpha_{1} \beta_{1}^{l-1}\right|^{2}+\left|\alpha_{2} \beta_{2}^{l-1}\right|^{2}=1$. In turn, by [11, Proposition 8.1.7(ii)], $S_{\boldsymbol{\lambda}}$ is quasinormal if and only if $\left|\beta_{1}\right|=\left|\beta_{2}\right|=1$. Set

$$
f(x)=\frac{\log \left(\frac{\log (2-x)}{-\log x}\right)}{\log \left(\frac{x}{2-x}\right)}, \quad x \in(0,1) .
$$

It is a simple matter to verify that $f(x)>0$ for all $x \in(0,1)$, and

$$
\lim _{x \rightarrow 0+} f(x)=0 .
$$

Hence, there exists $\gamma_{n} \in(0,1)$ such that $0<(n-1) f\left(\gamma_{n}\right) \leqslant 1$. A standard calculation shows that the function $g(x)=\gamma_{n}^{\frac{x}{n-1}}+\left(2-\gamma_{n}\right)^{\frac{x}{n-1}}$ is strictly 
increasing on $\left[(n-1) f\left(\gamma_{n}\right), \infty\right)$, and consequently on $[1, \infty)$. Now, taking $\alpha_{1}=\alpha_{2}=\left(\frac{1}{2}\right)^{\frac{1}{2}}, \beta_{1}=\gamma_{n}^{\frac{1}{2(n-1)}}$ and $\beta_{2}=\left(2-\gamma_{n}\right)^{\frac{1}{2(n-1)}}$, we verify that

$$
\begin{aligned}
\alpha_{1}^{2} \beta_{1}^{2(k-1)}+\alpha_{2}^{2} \beta_{2}^{2(k-1)} & =\frac{1}{2} g(k-1) \\
& \neq \frac{1}{2} g(n-1)=\alpha_{1}^{2} \beta_{1}^{2(n-1)}+\alpha_{2}^{2} \beta_{2}^{2(n-1)}=1
\end{aligned}
$$

whenever $k, n \geqslant 2$ and $k \neq n$, which means that $S_{\boldsymbol{\lambda}}$ satisfies (5.1) and is not quasinormal (the latter also follows from Lemma 3.5).

Concluding this section, we construct a quasinormal operator $C$ such that $C^{* n} \subsetneq\left(C^{n}\right)^{*}$ for every $n \geqslant 2$. Recall that, by Lemma 3.5, $C^{* n} C^{n}=$ $\left(C^{n}\right)^{*} C^{n}$ for every quasinormal operator $C$ and for all $n \in \mathbb{Z}_{+}$.

Example 5.6. Let $S$ be an isometry of multiplicity 1 on a complex Hilbert space $\mathcal{M}$ with a normalized cyclic vector $e_{0}$. Set $e_{n}=S^{n} e_{0}$ for $n \in \mathbb{N}$. Then

$$
S^{* k} e_{n}=\left\{\begin{array}{ll}
e_{n-k} & \text { if } k \leqslant n, \\
0 & \text { if } k>n,
\end{array} \quad k, n \in \mathbb{Z}_{+} .\right.
$$

Put $\mathcal{H}=\bigoplus_{j=0}^{\infty} \mathcal{M}_{j}$ with $\mathcal{M}_{j}=\mathcal{M}$ for all $j \in \mathbb{Z}_{+}$. Let $\left\{r_{j}\right\}_{j=0}^{\infty}$ be a sequence in $\mathbb{R}_{+}$. Set $C=\bigoplus_{j=0}^{\infty} r_{j} S$. By Proposition A.1(iii), the operator $C$ is quasinormal. It follows from Proposition A.1(i) that $C^{n}$ is densely defined and $\left(C^{n}\right)^{*}=\bigoplus_{j=0}^{\infty} r_{j}^{n} S^{* n}$ for every $n \in \mathbb{Z}_{+}$. This altogether implies that for every integer $n \geqslant 2$,

$$
\begin{aligned}
\mathcal{D}\left(\left(C^{n}\right)^{*}\right) & =\left\{\sum_{j \in \mathbb{Z}_{+}}^{\oplus} f_{j} \in \mathcal{H}: \sum_{j=0}^{\infty} r_{j}^{2 n}\left\|S^{* n} f_{j}\right\|^{2}<\infty\right\}, \\
\mathcal{D}\left(C^{* n}\right) & =\left\{\sum_{j \in \mathbb{Z}_{+}}^{\oplus} f_{j} \in \mathcal{H}: \sum_{j=0}^{\infty} r_{j}^{2 k}\left\|S^{* k} f_{j}\right\|^{2}<\infty \text { for } k=1,2, \ldots, n\right\} .
\end{aligned}
$$

Assume that $\sup _{j \in \mathbb{Z}_{+}} r_{j}=\infty$. Then there exists a sequence $\left\{t_{j}\right\}_{j=0}^{\infty} \subseteq \mathbb{R}_{+}$ such that

$$
\sum_{j=0}^{\infty} t_{j}^{2}<\infty \quad \text { and } \quad \sum_{j=0}^{\infty} t_{j}^{2} r_{j}^{2}=\infty .
$$

Hence, by (5.2) and (5.3), we see that $\left\{t_{j} e_{n-1}\right\}_{j=0}^{\infty} \in \mathcal{D}\left(\left(C^{n}\right)^{*}\right) \backslash \mathcal{D}\left(C^{* n}\right)$ for all integers $n \geqslant 2$. Since $C^{* n} \subseteq\left(C^{n}\right)^{*}$, this shows that $C^{* n} \subsetneq\left(C^{n}\right)^{*}$ for all integers $n \geqslant 2$.

\section{Acknowledgment}

A substantial part of this paper was written while the first and the third authors visited Kyungpook National University during the spring and autumn of 2013. They wish to thank the faculty and the administration of this unit for their warm hospitality. 
Open Access. This article is distributed under the terms of the Creative Commons Attribution License which permits any use, distribution, and reproduction in any medium, provided the original author(s) and the source are credited.

\section{Appendix A. Orthogonal Sums}

For sake of completeness we sketch the proof of the following facts (cf. [24]).

Proposition A.1. Assume that $\mathcal{H}=\bigoplus_{\omega \in \Omega} \mathcal{H}_{\omega}$ is the orthogonal sum of complex Hilbert spaces $\mathcal{H}_{\omega}$ and $C=\bigoplus_{\omega \in \Omega} C_{\omega}$ is the orthogonal sum of operators $C_{\omega}$ in $\mathcal{H}_{\omega}$. Then

(i) $C^{n}$ is densely defined and $\left(C^{n}\right)^{*}=\bigoplus_{\omega \in \Omega}\left(C_{\omega}^{n}\right)^{*}$ provided $n \in \mathbb{N}$ and $\overline{\mathcal{D}\left(C_{\omega}^{n}\right)}=\mathcal{H}_{\omega}$ for all $\omega \in \Omega$,

(ii) $C^{*} C=\bigoplus_{\omega \in \Omega} C_{\omega}^{*} C_{\omega}$ and $|C|=\bigoplus_{\omega \in \Omega}\left|C_{\omega}\right|$ provided $C_{\omega}, \omega \in \Omega$, are closed and densely defined,

(iii) $C$ is quasinormal if and only if $C_{\omega}$ is quasinormal for every $\omega \in \Omega$.

Proof. (i) It is well-known that (i) holds for $n=1$. Assume that $n \geqslant 2$. It is easily seen that $\mathcal{D}\left(\bigoplus_{\omega \in \Delta} C_{\omega}^{n}\right)=\bigoplus_{\omega \in \Delta} \mathcal{D}\left(C_{\omega}^{n}\right) \subseteq \mathcal{D}\left(C^{n}\right)$ for every finite nonempty set $\Delta \subseteq \Omega$. This implies that $C^{n}$ is densely defined. Since $C^{n} \subseteq \bigoplus_{\omega \in \Omega} C_{\omega}^{n}$, we deduce that $\bigoplus_{\omega \in \Omega}\left(C_{\omega}^{n}\right)^{*} \subseteq\left(C^{n}\right)^{*}$. To prove the reverse inclusion, take $g=\sum_{\omega \in \Omega}^{\oplus} g_{\omega} \in \mathcal{D}\left(\left(C^{n}\right)^{*}\right)$ with $g_{\omega} \in \mathcal{H}_{\omega}$. Then there exists $c \in \mathbb{R}_{+}$such that

$$
\left|\left\langle g, C^{n} f\right\rangle\right|^{2} \leqslant c\|f\|^{2}, \quad f \in \mathcal{D}\left(C^{n}\right) .
$$

Hence, $\left|\left\langle g_{\omega}, C_{\omega}^{n} f\right\rangle\right|^{2} \leqslant c\|f\|^{2}$ for all $f \in \mathcal{D}\left(C_{\omega}^{n}\right)$, which implies that $g_{\omega} \in \mathcal{D}\left(\left(C_{\omega}^{n}\right)^{*}\right)$ for every $\omega \in \Omega$. Applying (A.1) again, we see that

$$
\left|\left\langle\sum_{\omega \in \Delta}^{\oplus}\left(C_{\omega}^{n}\right)^{*} g_{\omega}, f\right\rangle\right|^{2}=\left|\left\langle g, C^{n} f\right\rangle\right|^{2} \leqslant c\|f\|^{2}, \quad f \in \mathcal{D}\left(\bigoplus_{\omega \in \Delta} C_{\omega}^{n}\right),
$$

which implies that $\sum_{\omega \in \Delta}\left\|\left(C_{\omega}^{n}\right)^{*} g_{\omega}\right\|^{2} \leqslant c$ for every finite nonempty set $\Delta \subseteq \Omega$. This yields $\sum_{\omega \in \Omega}\left\|\left(C_{\omega}^{n}\right)^{*} g_{\omega}\right\|^{2} \leqslant c$. Hence $g \in \mathcal{D}\left(\bigoplus_{\omega \in \Omega}\left(C_{\omega}^{n}\right)^{*}\right)$, which proves (i).

(ii) Clearly $C$ is closed and densely defined. Applying (i) with $n=1$, we get $C^{*} C \subseteq \bigoplus_{\omega \in \Omega} C_{\omega}^{*} C_{\omega}$. Hence, by the maximality of selfadjoint operators, we obtain the first equality in (ii). In view of (i), it is clear that the operator $\bigoplus_{\omega \in \Omega}\left|C_{\omega}\right|$ is positive and selfadjoint, and $\left(\bigoplus_{\omega \in \Omega}\left|C_{\omega}\right|\right)^{2} \subseteq \bigoplus_{\omega \in \Omega}\left|C_{\omega}\right|^{2}$. Using the maximality of selfadjoint operators and the first equality in (ii), we obtain the second one.

(iii) Suppose $C_{\omega}$ is quasinormal for every $\omega \in \Omega$. It follows from (ii) that

$$
E_{|C|}(\Delta)=\bigoplus_{\omega \in \Omega} E_{\left|C_{\omega}\right|}(\Delta), \quad \Delta \in \mathfrak{B}\left(\mathbb{R}_{+}\right) .
$$

Hence, by the inequality $\left\|E_{\left|C_{\omega}\right|}(\Delta)\right\| \leqslant 1$, we have

$$
E_{|C|}(\Delta) C \subseteq \bigoplus_{\omega \in \Omega} E_{\left|C_{\omega}\right|}(\Delta) C_{\omega} \subseteq \bigoplus_{\omega \in \Omega} C_{\omega} E_{\left|C_{\omega}\right|}(\Delta)=C E_{|C|}(\Delta), \quad \Delta \in \mathfrak{B}\left(\mathbb{R}_{+}\right),
$$


which shows that $C$ is quasinormal. The reverse implication is obvious because each $\mathcal{H}_{\omega}$ reduces $C$.

\section{References}

[1] Arveson, W.: A Short Course on Spectral Theory. Springer, New York (2002)

[2] Birman, M.Sh., Solomjak, M.Z.: Spectral Theory of Selfadjoint Operators in Hilbert Space. D. Reidel Publishing Co., Dordrecht (1987)

[3] Brown, A.: On a class of operators. Proc. Am. Math. Soc. 4, 723-728 (1953)

[4] Budzyński, P.: A note on unbounded hyponormal composition operators in $L^{2}$-spaces. J. Funct. Sp. Appl. 2012, 1-8. doi:10.1155/2012/902853

[5] Chernoff, P.R.: A semibounded closed symmetric operator whose square has trivial domain. Proc. Am. Math. Soc. 89, 289-290 (1983)

[6] Cichoń, D., Jung, I.B., Stochel, J.: Normality via local spectral radii. J. Oper. Theory 61, 253-278 (2009)

[7] Conway, J.B.: The theory of subnormal operators. In: Mathematical Surveys and Monographs, Providence, Rhode Island (1991)

[8] Daniluk, A.: On the closability of paranormal operators. J. Math. Anal. Appl. 376, 342-348 (2011)

[9] Embry, M.R.: A generalization of the Halmos-Bram criterion for subnormality. Acta Sci. Math. (Szeged) 35, 61-64 (1973)

[10] Istrăţescu, V., Saitô, T., Yoshino, T.: On a class of operators. Tôhoku Math. J. 18, 410-413 (1966)

[11] Jabłoński, Z.J., Jung, I.B., Stochel, J.: Weighted shifts on directed trees. Mem. Am. Math. Soc. 216(1017), viii+107pp (2012)

[12] Jabłoński, Z.J., Jung, I.B., Stochel, J.: A non-hyponormal operator generating Stieltjes moment sequences. J. Funct. Anal. 262, 3946-3980 (2012)

[13] Jabłoński, Z.J., Jung, I.B., Stochel, J.: A hyponormal weighted shift on a directed tree whose square has trivial domain. Proc. Am. Math. Soc. (in press)

[14] Jibril, A.A.S.: On operators for which $T^{* 2} T^{2}=\left(T^{*} T\right)^{2}$. Int. Math. Forum 46, 2255-2262 (2010)

[15] Kaufman, W.E.: Closed operators and pure contractions in Hilbert space. Proc. Am. Math. Soc. 87, 83-87 (1983)

[16] Majdak, W.: A lifting theorem for unbounded quasinormal operators. J. Math. Anal. Appl. 332, 934-946 (2007)

[17] Naimark, M.: On the square of a closed symmetric operator, Dokl. Akad. Nauk SSSR 26, 866-870 (1940); ibid. 28, 207-208 (1940)

[18] Ôta, S.: Some classes of $q$-deformed operators. J. Oper. Theory 48, 151$186(2002)$

[19] Rudin, W.: Real and Complex Analysis. McGraw-Hill, New York (1987)

[20] Stochel, J.: Lifting strong commutants of unbounded subnormal operators. Integr. Equ. Oper. Theory 43, 189-214 (2002)

[21] Stochel, J., Szafraniec, F.H.: On normal extensions of unbounded operators. II. Acta Sci. Math. (Szeged) 53, 153-177 (1989)

[22] Stochel, J., Szafraniec, F.H.: $\mathcal{C}^{\infty}$-vectors and boundedness. Ann. Polon. Math. 66, 223-238 (1997) 
[23] Stochel, J., Szafraniec, F.H.: Domination of unbounded operators and commutativity. J. Math. Soc. Jpn 55, 405-437 (2003)

[24] Stochel, J., Szafraniec, F.H.: Unbounded operators and subnormality, in preparation.

[25] Weidmann, J.: Linear Operators in Hilbert Spaces. Springer, Berlin (1980)

Zenon Jan Jabłoński and Jan Stochel $(\bowtie)$

Instytut Matematyki

Uniwersytet Jagielloński

ul. Łojasiewicza 6

30-348 Kraków, Poland

e-mail: Jan.Stochel@im.uj.edu.pl;

Zenon.Jablonski@im.uj.edu.pl

Il Bong Jung

Department of Mathematics

Kyungpook National University

Daegu 702-701, Korea

e-mail: ibjung@knu.ac.kr

Received: October 12, 2013.

Revised: February 4, 2014. 